\title{
CAECAL VOLVULUS WITH INTESTINAL MALROTATION: A CASE REPORT
}

\author{
Jayasuriya $\mathrm{KMGS}^{1}$, Gunawardena $\mathrm{DS}^{1}$ \\ Department of Medical Imaging, Royal Perth Hospital, Perth, Western Australia
}

Keywords:Caecal volvulus, Malrotation

DOI: http://doi.org/10.4038/sljr.v2i2.53

\section{Introduction}

Caecal volvulus is an infrequent cause of intestinal obstruction in adults. Surgical literature reports this as a rare acute presentation of intestinal malrotation in adults ${ }^{1}$. This is a case report of preoperative radiological diagnosis of uncomplicated caecal volvulus with underlyingmalrotation of intestine.

\section{Case Report}

A 46 year old female presented to the emergency department with generalized acute abdominal pain. She was haemodynamically stable but was found to be in moderate distress. She had no significant past medical history. Physical examination revealed a distended abdomen and generalized tenderness with a tympanic percussion tone in the central abdomen. Laboratory investigations were unremarkable.
There was no sub diaphragmatic free air in the erect chest radiograph. Supine abdominal radiograph showed a grossly dilated, solitary, comma shaped intestinal loop located in the central abdomen with preserved haustrations. No other dilated intestinal loops were seen (Figure 1a). Ultrasound scan revealed a trace of free fluid in the periumbilical region (Figure1b). Suspicion of a caecal volvulus was communicated to the clinical team at that point. After surgical referral an urgent contrast enhanced CT abdomen and pelvis was performed. It demonstrated a grossly distended and rotated caecum measuring 12 $\mathrm{cm}$ in transverse diameter, lying in the central abdomen. It contained air and faecal residue. Normal caecal wall enhancement was present without evidence ofpneumatosis coli or pneumoperitonium excluding wall ischemia or perforation. The associated "whirl sign" of the twisted mesentery with superior mesenteric artery and vein leading towards the dilated segment of intestines

Corresponding author: Jayasuriya KMGS, <sandeepani.jayasuriya@ gmail.com>

iD http://orcid.org/0000-0001-6625-778X 
was seen in the central abdomen lying to the left of the midline (Figures2 a,c). These features confirmed the diagnosis of uncomplicated caecal volvulus. On further review of $\mathrm{CT}$, there were non dilated small intestinal loops lying in the right side of the abdomen with a duodeno-jejunal junction located in the right sub hepatic area below the level of the first part of duodenum(Figure 2d,e). The large intestines distal to the caecum were collapsed and were seen largely on to the left side of abdomen. Superior mesenteric vein was seen anterior and to the left of the superior mesenteric artery in an altered anatomical relationship. (Figure2b) These findings raised the possibility of an under lying malrotation of intestines.

Emergency laparotomy confirmed the imaging diagnosis of caecal volvulus with intestinal malrotaion and right hemicolectomy and iliocolic anastomosis was performed. Post-operative complications of paralytic ileus and right basal pneumonia lengthened her hospital stay by a few days.

\section{Discussion}

Caecal volvulus accounts for less than $2 \%$ of all intestinal obstructions. It is usually found at a relatively younger age group between 30 to 60 years ${ }^{1}$. Contributing factors for this development include a mobile caecum on mesentery, pelvic mass, adhesions, thirdtrimester pregnancy, chronic constipation, ileus and distal obstructing lesion ${ }^{2}$.

Pathophysiology of caecal volvulus describes twisting of the caecum in the axial plane, rotating clockwise or counterclockwise around its long axis. (Figure 3a) Ectopic left upper quadrant position of caecum is seen, when associated with inversion. The terminal ileum can usually twist along with the ceacum and visualization of a gas-filled appendix will help to locate the caecum. There is a variant of caecal volvulus termed a "ceacal bascule", in which the ceacum does not twist but folds antero-medially along a fibrotic band, acting as a flap valve(Figure $3 b)^{1}$.

Midgut malrotation is a congenital anomaly that presents during the first year of life. The adult mid gut malrotation is either asymptomatic or present with chronic abdominal pain. HoweverMoldrem et al reported acute presentations of mid gut malrotation in adults with volvulus of the midgut or caecum ${ }^{3}$.

The basic plain radiograph of abdomen will illustrate a single dilated, comma or foetal shaped colonic loop located away from the right iliac fossa, containing a single air fluid level with preserved haustral markings. In contrast, sigmoid volvulus presents with two colonic limbs in an inverted $U$ appearance with two separate air-fluid levels and loss of haustral markings. However in $50 \%$ of cases with associated small intestinal obstruction, the ceacal volvulus may not give a clear abdominal $X$ - ray diagnosis. Contrast enema demonstrating a beak-like tapering at the level of the volvulus, could be a useful imaging modality in the absence of CT facilities.

$\mathrm{CT}$ as the imaging method of choice for intestinal obstruction will reveal a dilated and rotated caecum located anywhere in a line extending from RIF to left hypochondrium. It has the added benefit of early identification of potentially fatal complications, such as ischemia and perforation. Macari et al described the usefulness of the location of the "whirl sign" in differentiating this from a sigmoid volvulus where the "whirl" is on the right incaecalvolvulus and it's on the left in 
sigmoid volvulus. ${ }^{4}$ But this association was not proved here in our patient probably due to under lying intestinal malrotation. Furthermore, an absent "whirl sign"favours a caecalbascule ${ }^{2}$.

\section{Conclusion}

Caecal volvulus is a rare but imperative cause of intestinal obstruction in adults. Vigilance of an underlying intestinal malrotation is highlighted in this case report. CT with multiplanar reconstructions is vital, in prompt and accurate diagnosis of caecal volvulusand its complications prior to urgent surgical intervention.

Conflict of Interest: Authors declare that they do not have conflict of interest regarding this case report
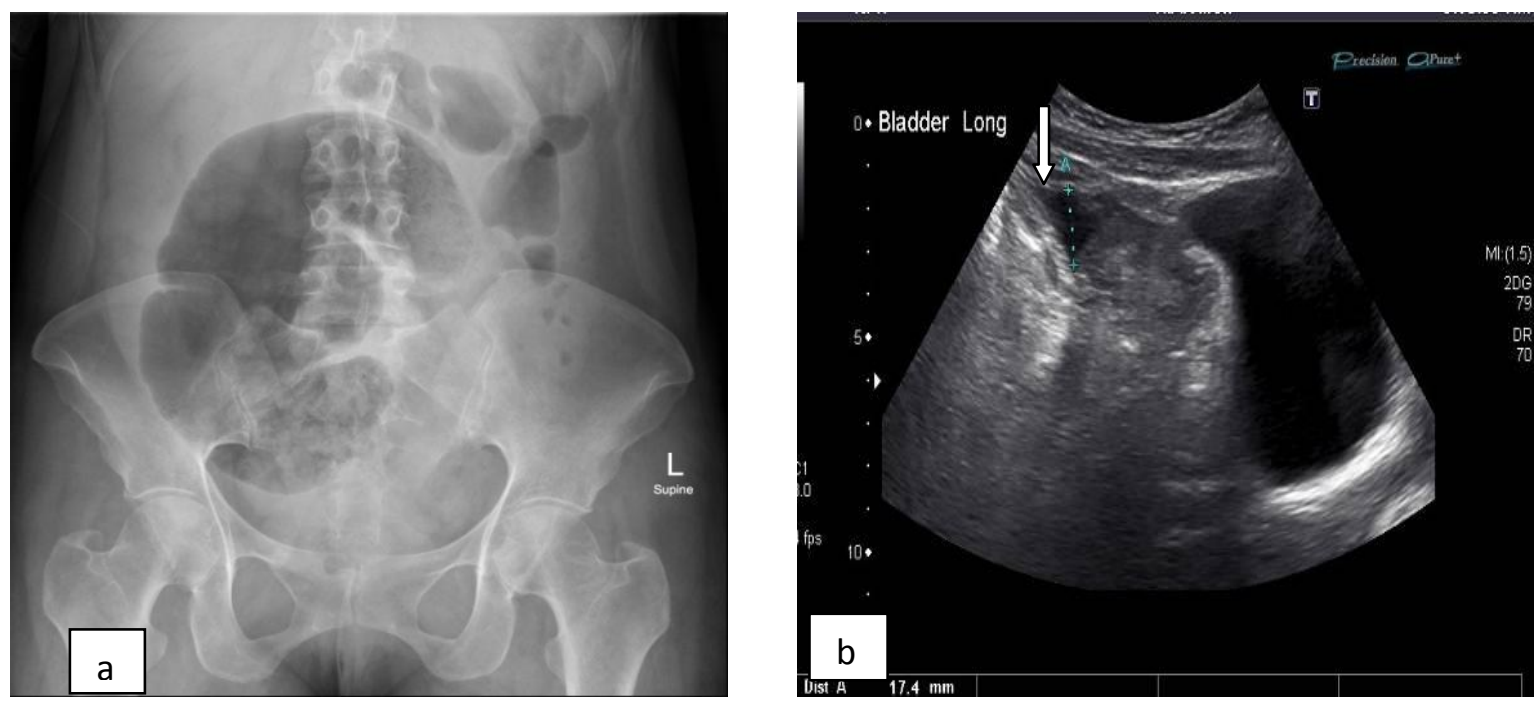

Figure 1: (a)Plain radiograph abdomen supine demonstrates a single dilated comma shaped bowel loop in the central abdomen with haustral markings (b)Ultrasound scan of the abdomen showing a trace of free fluid in the peri -umbilical region(arrow) 

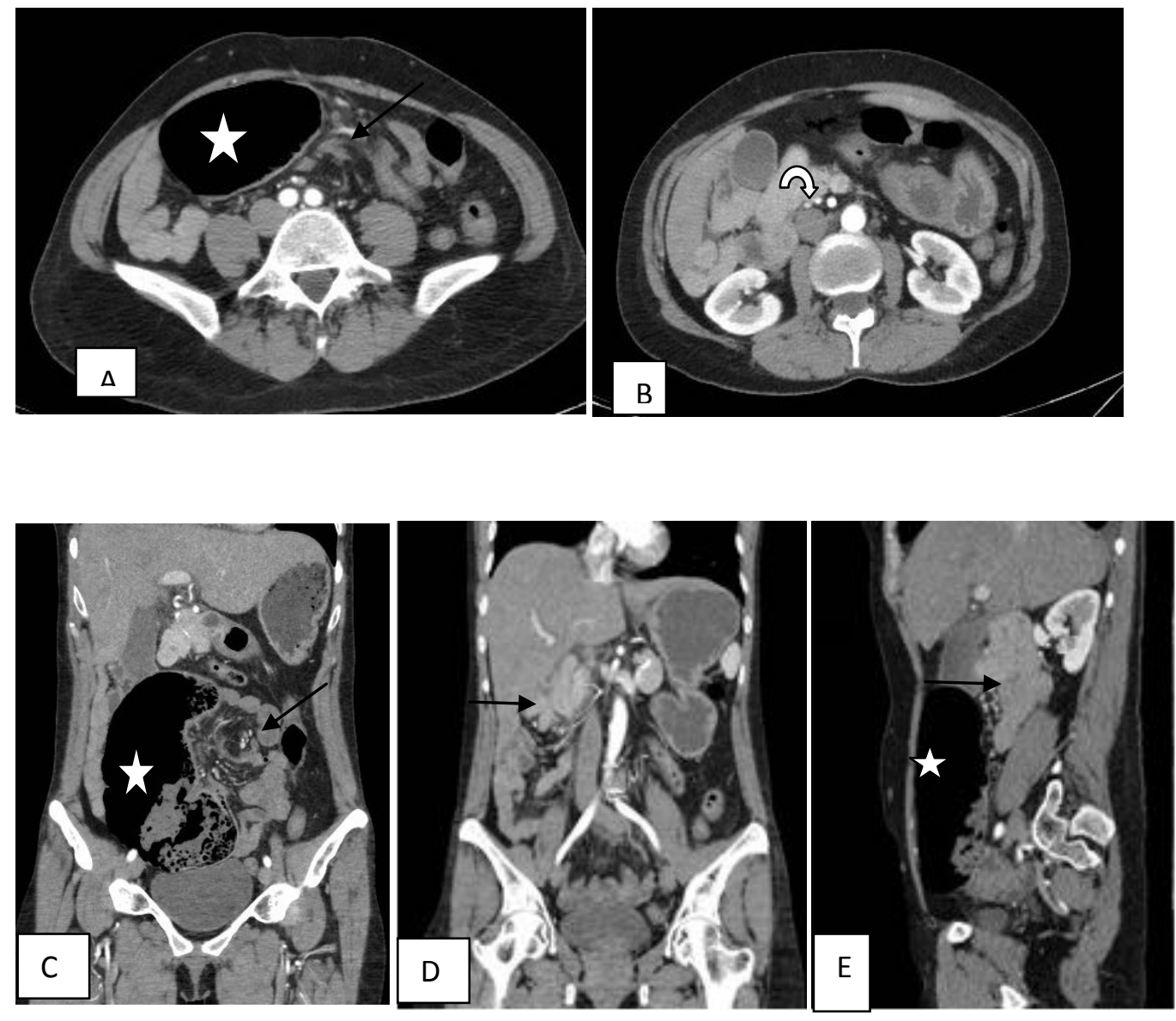

Figure 2: CT abdomen and Pelvis (A)Axial and (C)coronal CT images confirm the dilated caecum $(\hbar)$ with the twisted mesenteric vessels /Whirl sign $(\sim)$ pointing towards the caecum.Coronal (D) sagittal (E) CT images show the presence of small bowel in the right side of the abdomen with right sided duodeno-jejunal junction $(\rightarrow)$ and axial image $(B)$ shows the position of superior mesenteric vein anterior and left to the superior mesenteric( $($ ) artery 

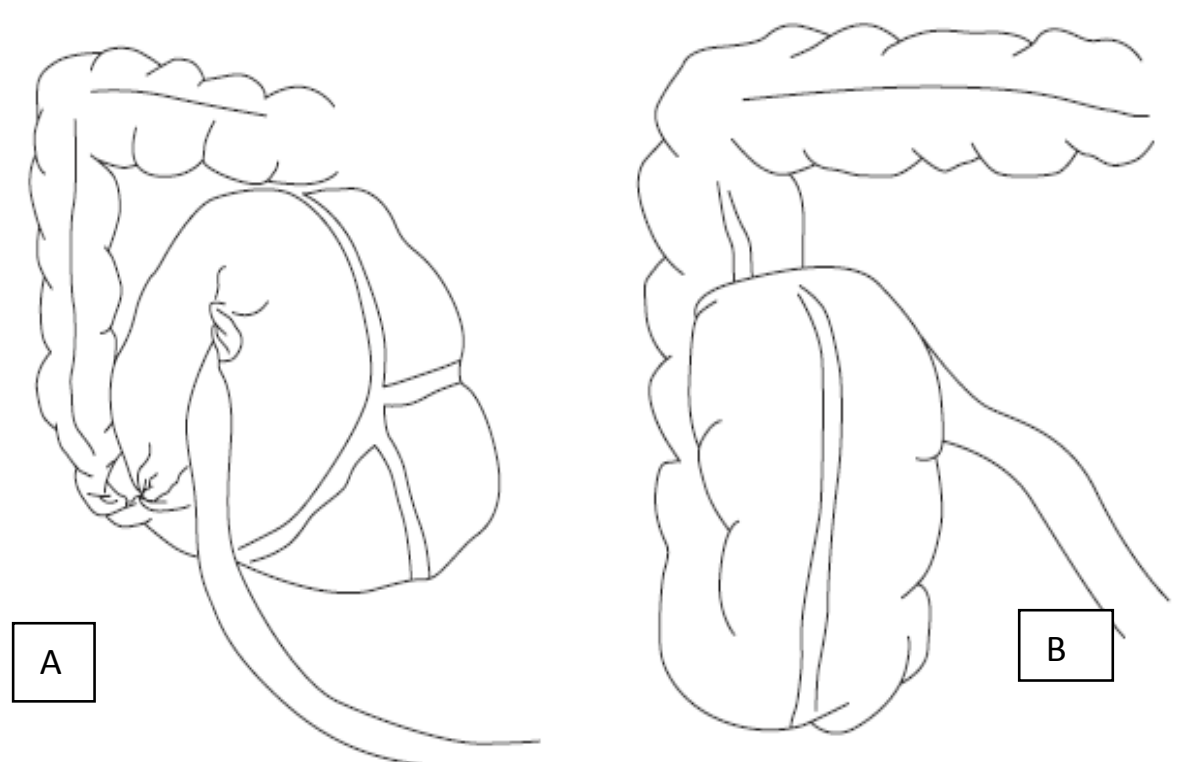

Figure 3:Schematic diagram of caecal volvulus showing axial twisting of the ascending colon and terminal ileum resulting in closed loop obstruction of the caecum in $A$. Schematic diagram of caecal bascule showing anterior folding of the caecum resulting in closed loop obstruction of the caecum in $\mathrm{B}^{1}$. 